\title{
Hemangioma of the Thoracic Spine Involving Multiple Adjacent Levels: Case Report
}

\author{
A. Blankstein, MD, R. Spiegelmann, MD, I. Shacked, MD, E. Schinder, \\ MD, A. Chechick, MD \\ Department of Neurosurgery, Department of Orthopaedic Surgery A, Department \\ of Pathology, Chaim Sheba Medical Center, Tel-Hashomer affiliated to the Sackler \\ School of Medicine, Tel-Aviv, Israel.
}

\section{Summary}

An unusual case of hemangioma involving three adjacent dorsal vertebrae with a clinical picture of progressive paraparesis is presented.

Radiological verification of the lesion can be expected if possible, using plain $X-$ Rays, tomography, $C T$ scan and myelography. The roentgenographic appearances of vertebral hemangioma were characteristic, but only $C T$ revealed the true extent of the disease.

Reports of such a case have appeared infrequently in the literature. [Karshner 1936, Bailey 1955, Reeves 1964, Robbins 1958]

Key words: Hemangioma; Thoracic spine; Multiple adjacent level spinal hemangioma.

\section{Introduction}

Hemangiomas of bone are slow growing benign lesions composed of masses of fully developed adult blood vessels. They occur frequently as silent lesions in any portion of the skeletal system and other organs (Turek, 1984). Hemangiomas of the vertebral column occur more frequently in women and at an age distribution between 12-76 years. Most lesions are asymptomatic and are discovered accidentally on X-ray by the typical appearance of prominent, thickened, vertical trabecular pattern. It is difficult to know how many of these silent lesions are true hemangiomas, because tissue is seldom obtained for histological examination. Toepfer (1928) examined 2154 spinal columns and found an incidence of $12 \%$ of angiomatous masses in the vertebrae. Junghanns (1932) found an incidence of $10 \%$ of vertebral angiomata in 10000 spine X-Rays. Probably, most of these, represent local varicosities and not true hemangiomas (Jaffe, 1958). Certainly clinical problems are rare, and require further investigation and treatment. We describe a symptomatic case with rare multilevel involvement. 


\section{Case report}

A 65-year-old woman was referred to the Neurosurgical Department of the Chaim Sheba Medical Center for evaluation of slowly progressive paraparesis of 3 years duration. Three months prior to admission she noticed weakness of the lower extremities which progressed so that she required to use a wheel chair. On examination a severe spastic paraparesis with bilateral Babinski responses was demonstrated. Touch, position, vibration and superficial pain sensations were diminished in both legs and up to the T10 dermatome. No hemangiomas were seen on the skin. On tomograms of the thoracic spine, typical parallel striations were demonstrated in the body of T10. (Figs 1,2)
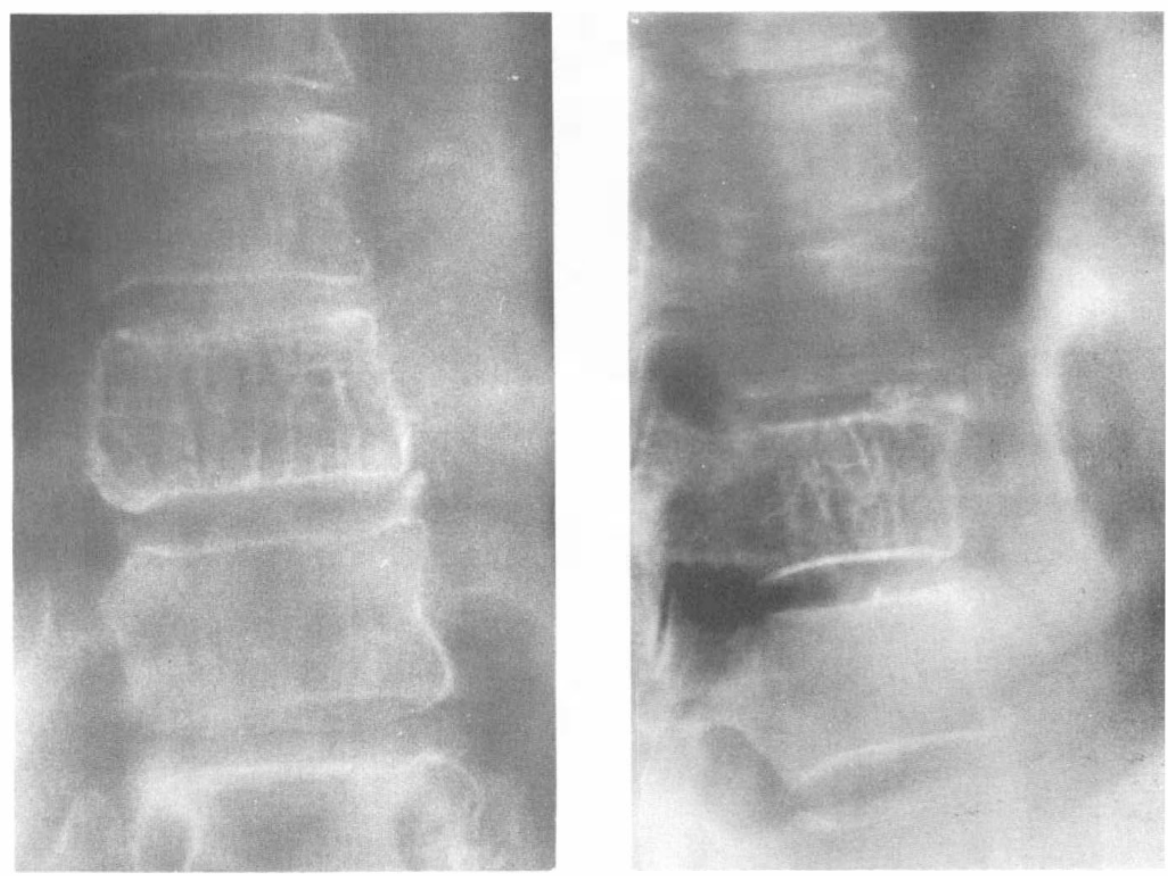

Figure 1,2 Tomography AP and lateral of T10 Typical parallel vertical trabeculation of the body extending to the posterior elements. Note at the lateral view the sharp sclerozis of the inferior end plate, suggesting a benign condition, and the invasion of the T9-T10 interspace by the lesion.

Axial CT demonstrated the typical appearance of the lesion in three adjacent different levels: T8-T9-T10. (Fig. 3 A-B, Fig. 4)

A myelogram revealed a complete block at T10 (Fig. 5) with the typical pattern of extradural compression. The patient was operated upon. A wide laminectomy of D8,9,10 being performed. The histological examination confirmed the diagnosis of hemangioma (Fig. 6). Brisk bleeding was encountered principally during the laminectomy of T10. An anteriorly situated soft tissue mass was discovered. An attempt at excision of this mass resulted in severe bleeding. The procedure was therefore abandoned.

Following operation the patient showed slight improvement of motor power in her lower limbs. She was referred to a rehabilitation center where a course of radiotherapy was instituted. Five months following surgery she was discharged home. The spastic paraparesis showed marked improvement and the patient could walk without help. One year later she remains asymptomatic with no sensory deficit. She has a spasticity of her gait. 


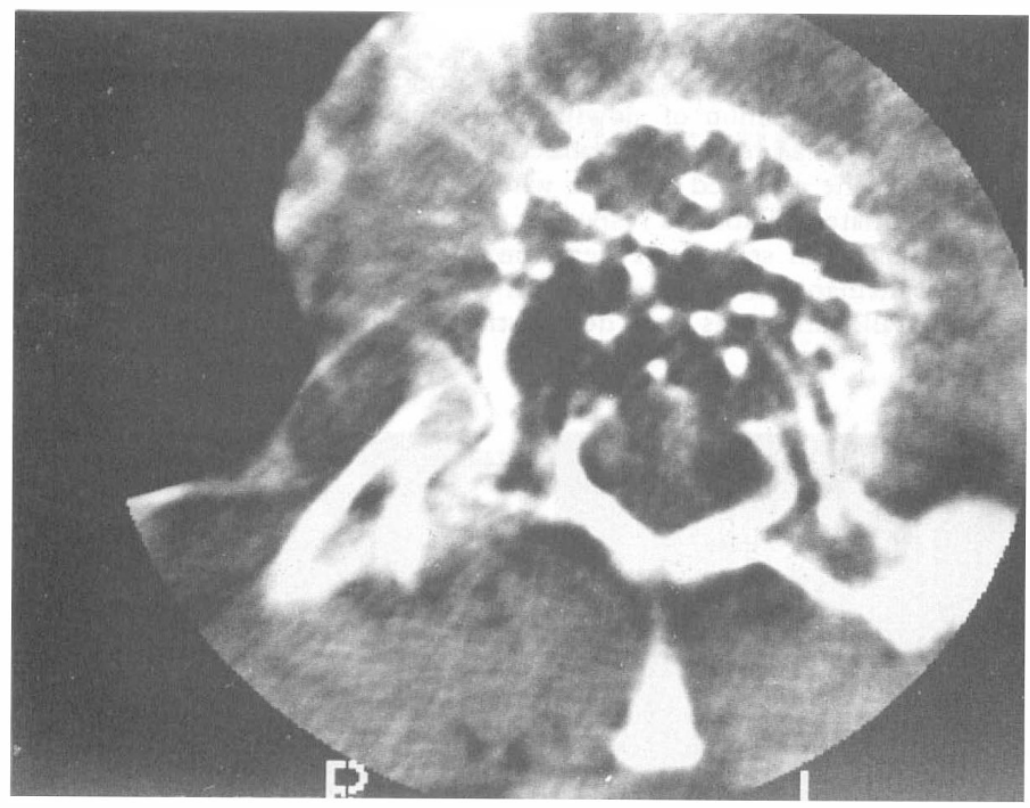

A

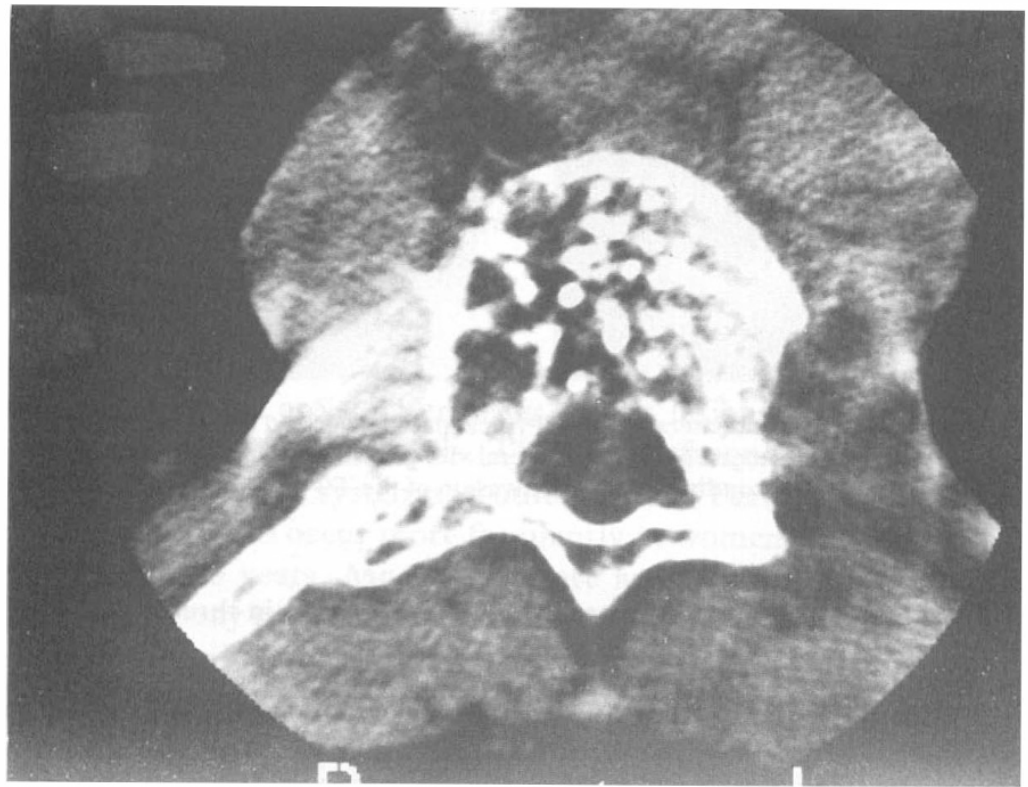

Figure 3 Axial CT (Non enhanced). (A) Note the striking appearance of T10 with its body and most of posterior elements destroyed by the lesion. The vertebral body is enlarged, dark wide spaces are separated by vertical, large trabeculae. The vertebral boundaries are irregular but mostly well defined. However, the anterior wall of the spinal canal is destroyed and soft tissue density is observed protruding from the body to the epidural space. (B) Cut through T9 shows better delineation of the body. The lesion invades the right transverse process. Note the sharp delineation of the lesion at the costotransverse joint. 


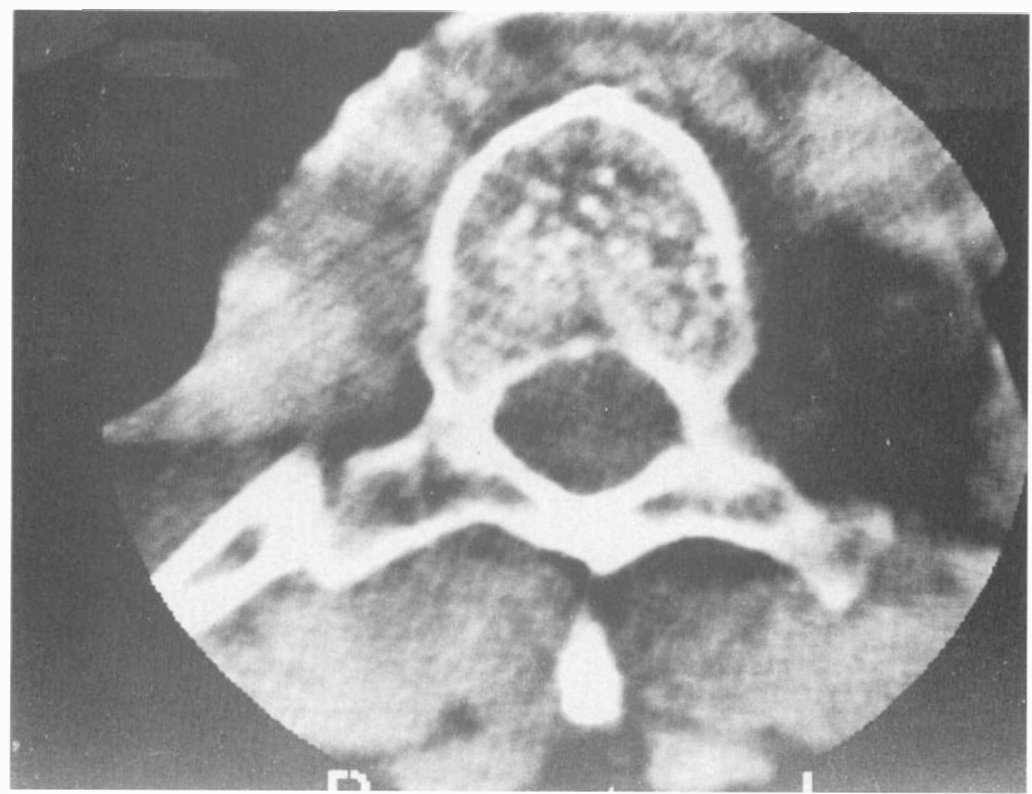

Figure 4 The body of T8 shows mild invasion by the lesion. The borders are sharply defined. The spinal canal looks normal.

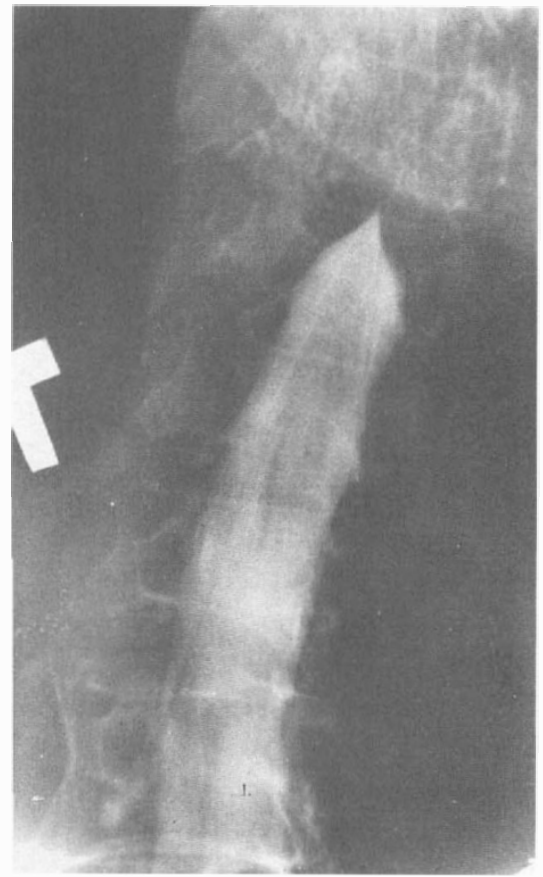

Figure 5 Metrizamide myelogram showing a complete block at T10 in the typical pattern of extradural compression. 


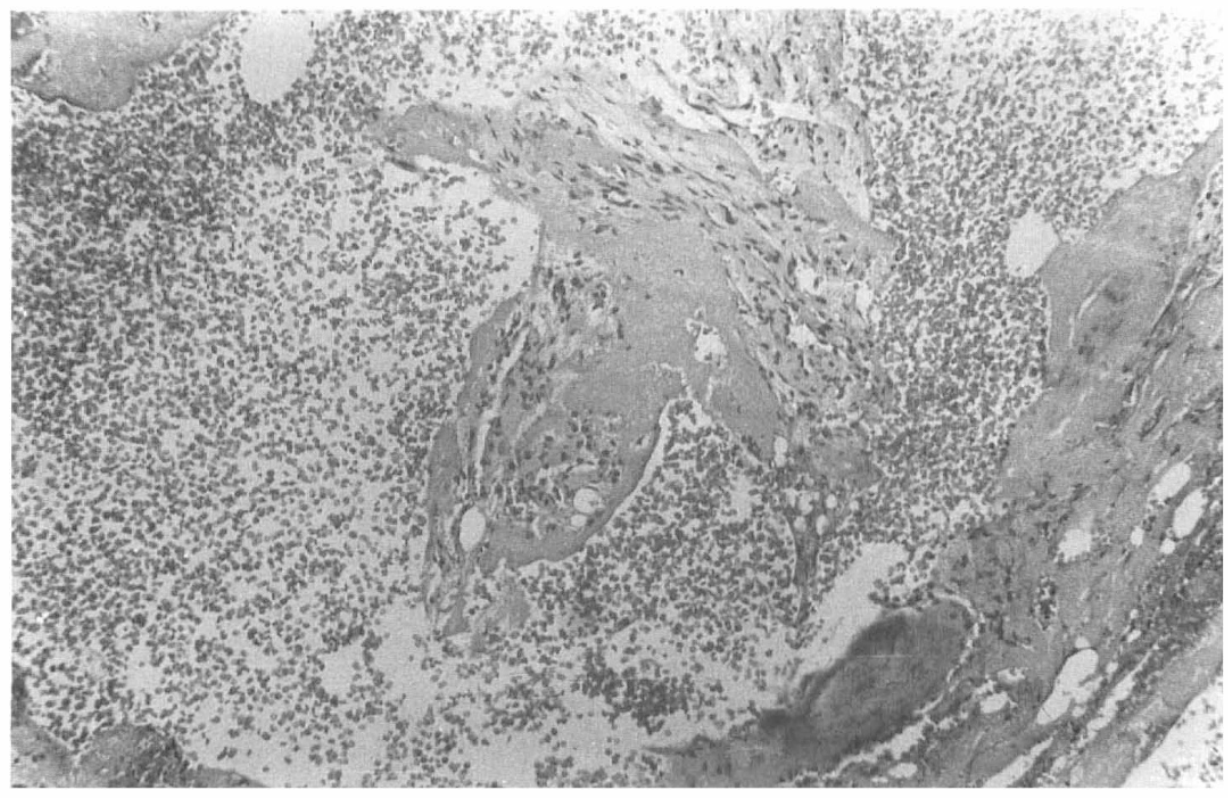

Figure 6 Microscopic description HE large irregular sinusoidal spaces filled with blood with bone spiculae, no osteoclastic activity is seen - and that is compatible with the diagnosis of a hemangioma.

\section{Discussion}

When hemangiomas become symptomatic they do so only because of pressure effect on the surrounding structures. In the case of hemangioma of vertebral column due to an expansile enlargement of bodies, pedicles and lamina or by direct invasion of the epidural space by the tumour (Bailey, 1929). In addition, when hemangioma destroys largely the vertebral body, a compression fracture may precipitate the onset of symptoms. Sometimes cutaneous hemangiomas of the skin occur in the same dorsal segment as the deep lesion, and may give a clue to diagnosis (Cobb, 1915). The radiographic findings are usually suggestive of a benign process when a fine shell of dense bone delimits the lesion. When this is the case the differential diagnosis from other benign processes like aneurysmal bone cyst or giant cell tumour is considered. Sometimes though, the margins are so poorly defined on X-ray, that a malignant tumour cannot be ruled out on the basis of plain X-rays. Myelography can reveal a pattern of extradural compression. In our case the use of computerised tomography CT revealed some striking features that deserve comment. While plain tomograms were able to show only the pathological process at T10, CT revealed that the disease had affected to a lesser extent the adjacent bodies of T8 and T9. In the pre-CT era Reeves (1964) reported a case of hemangioma involving the 2nd, 3 rd and 4 th thoracic vertebra. In his report, the multiple lesion was evident in plain X-rays. We failed to find other reports of multilevel vertebral hemangioma in our search of the English literature. Reports on CT of hemangioma are scarce. We believe that the CT appearance of hemangioma is so characteristic that confusion with other benign tumours of vertebra can be avoided. 
A honeycomb aspect is also encountered in aneurysmal bone cyst, but in the latter case the dark spaces are wider and the dense vertical trabeculae of hemangioma are lacking. Giant call tumour is an expansile soft tissue mass without trabeculation.

It is possible that with increasing experience multilevel hemangioma will become less rare. The presence of the disease in three adjacent vertebral levels in our case and that reported by Reeves suggests that the process extended to the neighbourhood through the disc interspace. Although we are unable to prove this possibility, the alternative explanation, a multifocal disease, seems less likely. It has been said that the ability to cross a vertebral interspace and involve adjacent vertebra is a unique characteristic of aneurysmal bone cyst and a few malignant bone tumours like chordoma (Beabout, 1979). In order to include hemangioma of the spine in the differential diagnosis, further experience with CT survey in patients affected with this process will be needed. In our case, we decided to perform only a palliative procedure, a decompressive laminectomy. We considered that the curative alternative: a triple corporectomy by a transthoracic approach with graft fusion and stabilisation by rods was too formidable for a 65-year-old patient with a benign and slow growing tumour. There is general agreement that for asymptomatic hemangioma no operative treatment is required. Posterior decompression and radiotherapy have been used for symptomatic tumours, mainly due to the severe bleeding that results when direct attack of the lesion is attempted. Selective spinal angiography with embolisation alone or as preoperative procedure before direct anterior approach to the lesion requires highly qualified personnel and equipment.

\section{References}

BAIley P, BUCy PC 1929 Cavernous Hemangioma of the Vertebrae. Fournal of American Medical Association 92:1748-1751.

Beabout JW, McLeod RA, Dahlin OC 1979 Benign tumors. Seminars in Roentgenology 14:3335.

BELL RL 1955 Hemangioma of a dorsal vertebra with collapse and compression myelopathy. fournal of Neurosurgery 12:570-576.

Совв S 1915 Hemangioma of the spinal cord associated with skin naevi of the same metamere. Annals of Surgery 62:641-649.

GHORMLEY RK, ADSON AW 1941 Hemangioma of vertebrae fournal of Bone and foint Surgery 23:887-895.

JAFFE HL 1958 Tumors and Tumourous conditions of the Bones and Joints. Philadelphia, Lea \& Febiger, p224-225.

JunGHANNS J 1932 Haemengiom des Brustwirbelkoerpers mit Rueckenmarkkompression. Laminektomie. Heilung. Arch. Klin. Chir. 169: 321-330.

KARSHNER RG, RAND CW, REEVES DL 1964 Epidural Hemangioma associated with hemangioma of the vertebrae Report of a case. Archives of Surgery 39:942-951.

REEVES DL 1964 Vertebral Hemangioma with compression of the spinal cord. Fournal of Neurosurgery 21:710-712.

RoBbINS LR, Fountain EM 1958 Hemangioma of cervical vertebras with spinal cord compression New England fournal Medicine 258:685-687.

TOEPFER D 1928 Ueber ein infiltrierend wachsendes Haemangiom der Haut und multiple Kapillarektasien der Haut and innerer Organe. II Zur Kenntnis der Wirbelangiome. 36:337345.

TUREK LS 1984 Orthopaedics Principles and Their Application. Philadelphia, Lippincott p655-657. 